\title{
Applying the data fusion method to evaluation of the performance of two control signals in monitoring polarization mode dispersion effects in fiber optic links
}

\author{
M. Dashtbani Moghari \\ dashtbani.2009@gmail.com \\ P. Rezaei \\ prezaei@semnan.ac.ir
}

A. Habibalahi

a.habibalahi@gmail.com
Department of Electrical and Computer Engineering, Semnan University, Semnan, Iran

Department of Electrical and Computer Engineering, Semnan University, Semnan, Iran

School of Mechanical Engineering, Iran University of Science and Technology, Tehran, Iran

With increasing distance and bit rate in fiber optic links the effects of polarization mode dispersion (PMD) have been highlighted. Since PMD has a statistical nature, using a control signal that can provide accurate information to dynamically tune a PMD compensator is of great importance. In this paper, we apply the data fusion method with the aim of introducing a method that can be used to evaluate more accurately the performance of control signals before applying them in a PMD compensation system. Firstly, the minimum and average degree of polarization $\left(D O P_{\min }\right.$ and $D O P_{\text {ave }}$ respectively) as control signals in monitoring differential group delay (DGD) for a system including all-order PMD are calculated. Then, features including the amounts of sensitivity and ambiguity in DGD monitoring are calculated for NRZ data format as DGD to bit time (DGD/T) varies. It is shown that each of the control signals mentioned has both positive and negative features for efficient DCD monitoring. Therefore, in order to evaluate features concurrently and increase reliability, we employ data fusion to fuse features of each control signal, which makes evaluating and predicting the performance of control signals possible, before applying them in a real PMD compensation system. Finally, the reliability of the results obtained from data fusion is tested in a typical PMD compensator.

[DOI: http://dx.doi.org/10.2971/jeos.2015.15008]

Keywords: Data fusion, decision making, polarization mode dispersion (PMD), degree of polarization (DOP), differential group delay (DGD)

\section{INTRODUCTION}

Increasing bit rate and distance in fiber optic links have made polarization mode dispersion (PMD) a serious problem in high-speed transmission at $40 \mathrm{~Gb} / \mathrm{s}$ or beyond. PMD is a statistically arbitrary pulse distortion that arises from the random birefringence in optical fibers and limits pulse width and linearity. Generally, it is studied in a Taylor series expansion framework in terms of the first-order PMD, second-order PMD, etc. Furthermore, with the increases in the bandwidth of telecommunication systems, the effects of higher order PMD have become more highlighted. Therefore, the investigation of higher order PMD compensation (PMDC) and higher order PMD measurement techniques have been more prominent recently [1]-[5].

Moreover, since PMD has a statistical nature and it causes a delay known as differential group delay (DGD) between the two principle states of polarization (PSP), the instantaneous DGD should be monitored in a PMDC system in order to evaluate dynamically the quality of signals affected by PMD and provide PMDC with a control signal. Different monitoring signals are applied in PMDC systems such as degree-ofpolarization (DOP) [6], radio-frequency (RF) spectrum of the received signal, the eye-opening (EO) penalty, the phase difference of a given frequency component after PSP filtering [7] - and so forth. There are some advantages to using the DOP as monitoring signal, as it is simple, bit-rate independent and does not require high-speed electronics [8].

Generally, PMDC compensators are divided into two groups: electronic compensators and optical compensators: each of which has merits and demerits. For instance, it is simple to include electronic PMD compensators in line-terminals - and they are cost-effective, operate at high speed and are FEC compatible. But their dependence on modulation format, IC technology capability and their operation on a single channel are significant disadvantages. Conversely, optical compensators operate independently of bit rate and modulation format - and they are capable of compensating more channels concurrently. But they have lower speed and involve more complex feedback signal processing within the control algorithm [9].

What is crucial in an optical PMDC system is selecting a suitable control signal that can track PMD effects properly. When a single feature of the control signals is considered, it is readily possible to evaluate and compare different control signals together. But, in order to select more accurate control signals, two or more features should be evaluated and compared. In this case, a control signal may seem efficient in one aspect, while it is ineffective in other aspects. In this situation, selecting a more efficient control signal would be a challenge. In such circumstances, as we shall show in this paper, data fu- 
sion is a useful method that helps to provide a proper analysis of the different features of control signals concurrently and give the best interpretation of their performance -and, therefore, give accurate selection. In fact, data fusion is a strategy for integrating several database and knowledge sources -and it results in producing more accurate information than the initial data sources. In addition to analyzing data concurrently, increasing reliability of information can be achieved by data fusion.

In the present study, while there is all-order PMD, the minimum degree of polarization $\left(D O P_{\text {min }}\right)$ and average degree of polarization $\left(D O P_{a v e}\right)$ are considered and calculated as control signals in a PMD monitoring system with a polarization scrambler at the input of the fiber. Then, two features, including the amount of sensitivity and the amount of standard deviation (determining the amount of ambiguity) in DGD monitoring are obtained as the DGD-to-bit-time ratio (DGD/T) varies. It is shown that using $D O P_{\text {min }}$ as a control signal has the advantage of giving greater sensitivity to DGD/T variations and the disadvantage of giving greater ambiguity in DGD monitoring - rather than applying $D O P_{\text {ave }}$. As a result, decision making on the selection of a more efficient control signal and evaluating and comparing $D O P_{\min }$ and $D O P_{\text {ave }}$ is challenging, since each has merits and demerits. In fact, it is beneficial to take advantage of both of the control signals introduced. As a result, a data fusion approach has been used, since it is a method that integrates multiple data and knowledge and represents the same real-world object in a consistent, useful, and accurate representation, in order to improve the reliability of information obtained from various data sources. This technique of fusing information from different sources can also be used in electronic signal processing [10]. But, our focus in this paper is on using this method in optical domain PMD monitoring. The key aim of utilizing data fusion is to generate a combined result that offers the most exhaustive and reliable information feasible. Moreover, fusing various data sources together results in a more effective data representation [10].

Finally, for testing results achieved by data fusion method, $D O P_{\min }$ and $D O P_{\text {ave }}$ are employed in a simple feed-forward first-order (first term of Taylor series) PMDC and their effectiveness in lowering the outage probability is compared with results obtained from the data fusion method.

\section{PRINCIPALS AND METHODS}

\subsection{DOP Calculations in a System including first and all-order PMD}

In a DGD monitoring system based on the DOP (Figure 1), a polarization controller (PC) should be located at the input of the fiber-optic link, so as to make the input statesof-polarization (SOPs) become scrambled over the Poincare sphere, before launching light into the fiber. Furthermore, a polarimeter is used to measure the SOPs and specify the corresponding DOPs at the end of the link. The Jones transfer matrix of the fiber link (in the absence of polarization dependent

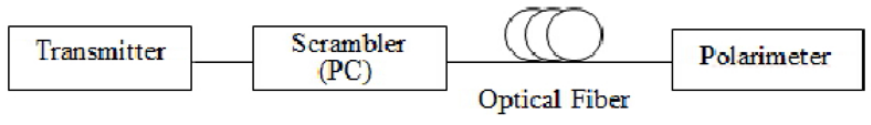

FIG. 1 A DGD monitoring system based on DOP.

loss) can be modeled by $[12,13]$

$$
T(\omega)=e^{-(\alpha(\omega) L+j \bar{\beta}(\omega) L)} U(\omega)
$$

Where $\alpha(\omega), \bar{\beta}(\omega)$ and L are the attenuation, the mean propagation constant, and the length of the fiber, respectively. Also, $\mathrm{U}(\omega)$ is the unitary matrix given by:

$$
U(\omega)=\left[\begin{array}{cc}
u_{1}(\omega) & u_{2}(\omega) \\
-u_{2}^{*}(\omega) & u_{1}^{*}(\omega)
\end{array}\right]
$$

With $\left|u_{1}(\omega)\right|^{2}+\left|u_{2}(\omega)\right|^{2}=1$. By using the analytical model given in reference [11], the effects of all-order PMD can be simulated and evaluated in an optical link. In this model, a PMD $\operatorname{vector}(\vec{\Omega})$ with the constant magnitude $\left(\Delta \tau_{0}=\left.\Delta \tau\right|_{\omega}=0\right)$ rotates on a circumference in the Stokes space with angular velocity $p$ written as:

$$
\vec{\Omega}=\left(-\Delta \tau_{0} \cos (p \omega),-\Delta \tau_{0} \sin (p \omega), 0\right)
$$

With this assumption, the coefficients of the Jones matrix become:

$$
\begin{aligned}
u_{1}(\omega)= & {\left[p \sin \left(\frac{p}{2} \omega\right) \sin \left(\frac{a}{2} \omega\right)+a \cos \left(\frac{p}{2} \omega\right) \cos \left(\frac{a}{2} \omega\right)\right.} \\
& \left.+j \Delta \tau_{0} \cos \left(\frac{p}{2} \omega\right) \sin \left(\frac{a}{2} \omega\right)\right] \frac{1}{a} \\
u_{2}(\omega)= & -\left[-p \cos \left(\frac{p}{2} \omega\right) \sin \left(\frac{a}{2} \omega\right)+a \sin \left(\frac{p}{2} \omega\right) \cos \left(\frac{a}{2} \omega\right)\right. \\
& \left.-j \Delta \tau_{0} \sin \left(\frac{p}{2} \omega\right) \sin \left(\frac{a}{2} \omega\right)\right] \frac{1}{a}
\end{aligned}
$$

Where $a=\sqrt{\Delta \tau_{0}^{2}+p^{2}}$, and where it is supposed that the output PSPs are aligned to the input PSPs at $\omega=0$.

Also, the Jones transfer matrix of the PC is defined as [13]:

$$
P=\left[\begin{array}{cc}
\cos (\theta) e^{j \varphi} & -\sin (\theta) \\
\sin (\theta) & \cos (\theta) e^{-j \varphi}
\end{array}\right]
$$

Where $\theta$ and $\varphi$ are angles that vary with time and describe the instantaneous SOP.

To scramble the input SOP over the Poincare sphere, a PC is used at the input of the optical link. Therefore, the total unitary matrix of the link will be [14]:

$$
U_{\text {tot }(\omega)}=U(\omega) P=\left[\begin{array}{cc}
\overline{u_{1}}(\omega) & \overline{u_{2}}(\omega) \\
-\bar{u}_{2}^{*}(\omega) & \bar{u}_{1}^{*}(\omega)
\end{array}\right]
$$

Where

$$
\begin{aligned}
& \overline{u_{1}}(\omega)=u_{1}(\omega) \cos (\theta) e^{j \varphi}+u_{2}(\omega) \sin (\theta) \\
& \overline{u_{2}}(\omega)=u_{2}(\omega) \cos (\theta) e^{-j \varphi}-u_{1}(\omega) \sin (\theta)
\end{aligned}
$$

Moreover, the Stokes vector definition of the instantaneous DOP of the scrambled signal after passing the polarimeter at 
the end of the optical link is [13]

$$
\operatorname{DOP}(\theta, \varphi)=\frac{\sqrt{\left(S_{1 \text { out }}\right)^{2}+\left(S_{2 o u t}\right)^{2}+\left(S_{3 o u t}\right)^{2}}}{\left(S_{0 o u t}\right)^{2}}
$$

And $S_{0 o u t}$ (the output signal optical power) is:

$$
S_{0 o u t}=\int_{-\infty}^{+\infty} S_{\text {out }}(\omega) d \omega
$$

Where $S_{\text {out }}(\omega)$ is the output signal optical power spectrum. Furthermore, output Stokes parameters, $S_{1 \text { out }}(\omega)$, $S_{2 o u t}(\omega)$ and $S_{3 o u t}(\omega)$ have been calculated in reference [13]. When there is all-order PMD, the instantaneous output Stokes parameters of the scrambled signal measured by a polarimeter can be calculated by replacing the coefficients of the unitary matrix in the Stokes parameters expressions in [13] with the coefficients in (6), while $u_{1}$ and $u_{2}$ in (7) should be replaced with expressions in (4). After calculating the instantaneous output Stokes parameters of the scrambled signal using the approach already described, the corresponding DOP can be obtained [14].

For calculation of $D O P_{\text {ave }}$, the following definition is used:

$$
D O P_{\text {ave }}=\sqrt{E\left\{D O P^{2}\left(\theta_{i}, \varphi_{i}\right)\right\}}
$$

Where $\theta_{i}$ and $\varphi_{i}$ are random variables with uniform distributions in the range [0 to $\left.\frac{\pi}{2}\right]$ and [0 to $\left.2 \pi\right]$. The notation $E\{\bullet\}$ also, indicates statistical averaging as:

$$
E\{\bullet\}=\frac{1}{2 \pi} \int_{0}^{2 \pi} \int_{0}^{\frac{\pi}{2}}(\bullet) \sin (2 \theta) d \theta d \varphi
$$

As a result, under the condition of all-order PMD, DOP ave can be achieved by applying (8) and substituting calculated output stokes parameters of the scrambled signal in it and then, employing (10) and (11). Additionally, when all-order PMD is present, $D O P_{\min }$ can be obtained by calculating (8) in various SOPs that uniformly cover the whole Poincare sphere - and then choosing a minimum sample [14].

Under the condition of first-order PMD being present - and assuming alignment of fast PSP with the SOP corresponding to $\left[\begin{array}{ll}1 & 0\end{array}\right]^{T}$ - the unitary transform matrix can be simplified to:

$$
U(\omega)=\left[\begin{array}{cc}
e^{j \omega D G D / 2} & 0 \\
0 & e^{-j \omega D G D} / 2
\end{array}\right]
$$

Then, when there is first-order PMD, for calculating $D O P_{\text {ave }}$ the coefficients of (12) are inserted into the coefficients of the unitary matrix in the calculated instantaneous output Stokes parameters of the scrambled signal expressions and then - by using (8), (10) and (11) - DOP ave can be obtained as:

$$
D O P_{\text {ave }}=\sqrt{\frac{1}{3}+\frac{2\left(\int_{-\infty}^{+\infty} \cos (\omega D G D) S_{\text {out }}(\omega) d \omega\right)^{2}}{3 S_{0 o u t}^{2}}}
$$

Furthermore, by replacing the expressions obtained for the instantaneous output Stokes parameters of the scrambled signal in (8) and, by applying Eq. (12), the instant DOP in the presence of first-order PMD is obtained as:

$$
=\sqrt{\cos ^{2}(2 \theta)+\sin ^{2}(2 \theta) \frac{\left(\int_{-\infty}^{+\infty} \cos (\omega D G D) S_{\text {out }}(\omega) d \omega\right)^{2}}{S_{0 \text { out }}^{2}}}
$$

Therefore, under the condition of first-order PMD, $D O P_{\min }$ is obtained by minimizing Eq. (14) with respect to $\theta$, as described in reference [13]

$$
D O P_{\min }=\frac{\int_{-\infty}^{+\infty} \cos (\omega D G D) S_{\text {out }}(\omega) d \omega}{S_{0 o u t}}
$$

\subsection{DGD monitoring based on DOP in Presence of all-order PMD}

To simulate the effects of PMD on DOP in the system shown in Figure 1, the model in [11] is used and pseudo-random bit sequences (PRBS) of super-Gaussian NRZ pulses with a word length of $2^{5}-1$ are launched in a $40-\mathrm{Gb} / \mathrm{s}$ transmission system. Then, $D O P_{\text {min }}$ and $D O P_{\text {ave }}$ are computed for $10^{4}$ fiber realizations generated by a random angular velocity $(\mathrm{p})$ of PSP with mean square value of $\pi D G D^{2} / 6$ and applying probability distribution function in [15]. Then, the mean and standard deviation of $D O P_{\min }$ and $D O P_{\text {ave }}$ are obtained.

The results are shown in Figures 2 and 3. Figure 2 shows $D O P_{\min }$ and $D O P_{\text {ave }}$ variations versus $\mathrm{DGD} / \mathrm{T}$ variations. By considering this Figure, it can be concluded that $D O P_{\min }$ is a more sensitive control signal in monitoring DGD/T variations compared with $D O P_{\text {ave }}$ since the slope of the minimum DOP vs. DGD/T curve ( $\triangle \mathrm{MDD}$ ) is more than the slope of the average DOP vs. DGD/T curve ( $\triangle \mathrm{ADD})$. The greater sensitivity of $D O P_{\min }$ to DGD/T variations is an advantage of employing it in a DGD monitoring system, as compared with employing $D O P_{\text {ave }}$. Moreover, the amount of ambiguity in monitoring DGD can be expressed by the standard deviation of DOP for each DGD value. As a result, by considering Figure 3 it can be concluded that when $D O P_{\min }$ is employed in a DGD monitoring system, the ambiguity in monitoring DGD is more than when $D O P_{\text {ave }}$ is used. The greater ambiguity in monitoring DGD is a disadvantage of using $D O P_{\min }$ in a DGD monitoring system compared with using DOP ave. Furthermore, as Figure 3 shows, the slope of Standard deviation of Minimum DOP vs. DGD/T curve $(\triangle \mathrm{SMDD})$ is more than the slope of Standard deviation of Average DOP vs. DGD/T curve $(\triangle \mathrm{SADD})$. In other words, when $D O P_{\min }$ is applied for DGD monitoring, the ambiguity of DGD monitoring increases faster. It can therefore be concluded from Figures 2 and 3 that using $D O P_{\text {min }}$ for monitoring DGD has the advantage of greater sensitivity against DGD/T variations and the disadvantage of greater ambiguity in DGD Monitoring. However, the performance of $D O P_{\text {ave }}$ is vice versa: it has less sensitivity (as its negative aspect) and less ambiguity (as its positive aspect) in monitoring DGD. Hence, determining the more efficient control signal in a DGD monitoring system is challenging because each of the control signals has a negative and a positive aspect, in order to be efficient. In this situation, using the data fusion method can be very helpful, owing to the fact 


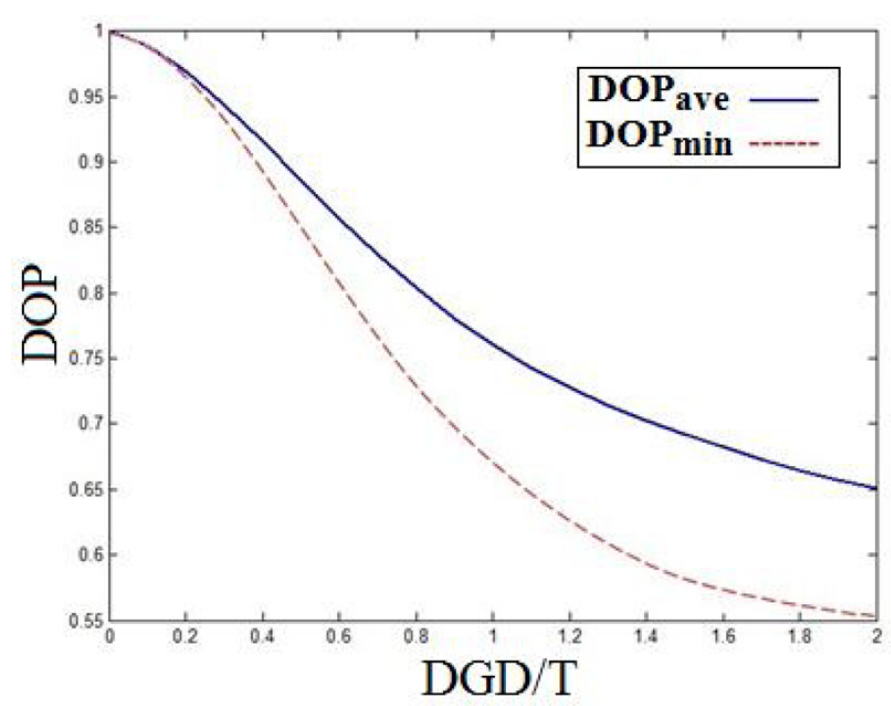

FIG. $2 D O P_{\min }$ and $D O P_{\text {ave }}$ variations versus $D G D / T$ variations.

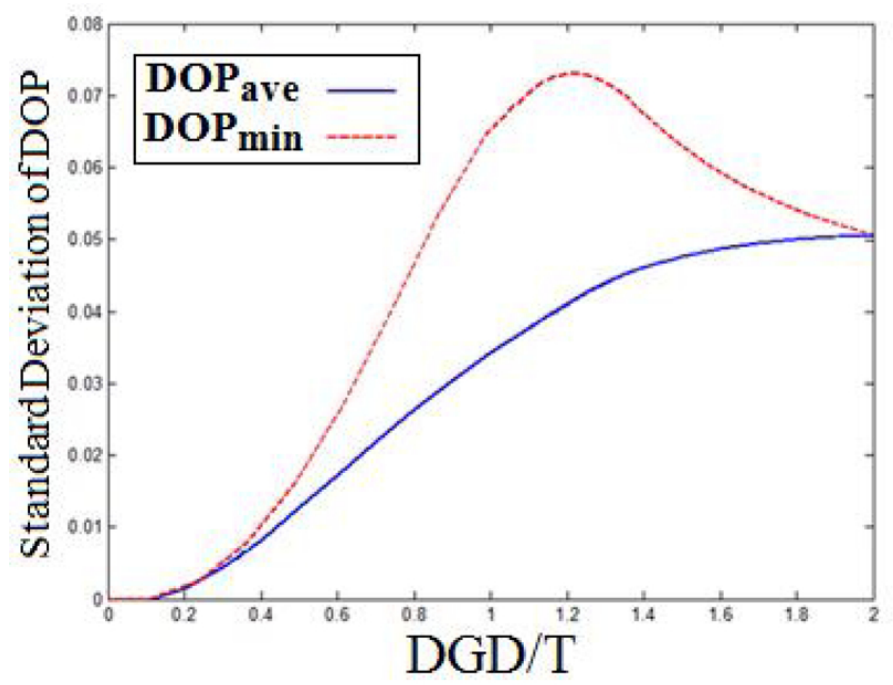

FIG. 3 Standard Deviation of $D O P_{\min }$ and $D O P_{\text {ave }}$ variations versus $D G D / T$ variations.

that, by this method, two features of each control signal (the amount of sensitivity and the ambiguity in monitoring DGD) are combined to assist us in the evaluation of two features of each control signal concurrently. In this way, we can achieve the best interpretation of the way these two control signals perform while considering both their negative and positive aspects in a DGD monitoring system, before employing them in a PMDC system.

\subsection{Data Fusion Method}

Data fusion is an approach to enhancement of information that results in obtaining a precise inference. In other words, in order to have accurate, all- inclusive and more reliable data, scholars use integrated information from different sources. As a result, data fusion assists researchers in possessing comprehensive analysis and decisions. Although data fusion has various manners, in this study the theory of fuzzy combination is used since this approach has a promising performance when the information contains, similar to our data, uncertainty and some extend of ambiguity $[10,16]$. In this study, two features of two control signals based on DOP (the amount of sensitivity and ambiguity in monitoring DGD) are employed to specify the more efficient control signal in a DGD monitoring system.

Zadeh [17] was the first scientist who generalized the classical concept of the Boolean logic and presented the fuzzy logic concept. In fuzzy logic, variables take a value ranging between 0 and 1 . In fact, the fuzzy logic was developed to deal with the introduction of partial truth in mathematics. That is, the truth value can vary between 0 as the completely false and 1 as the completely true. A fuzzy set is totally characterized using membership function. That is, a membership function for a fuzzy set ' $A$ ' on the universe of discourse ' $U$ ' is defined as:

$$
\mu_{A}(X): U \rightarrow[0,1]
$$

Decider, the person who wants to estimate or judge the outcome of the system, holds scope including translation and combination mathematically and utilizes a decision making function using various criteria; In fact, decider has the aim of look at his object from various angels providing different information from different aspects of the object in order to have an all- inclusive idea regarding the issue. As a result, a particularly desirable situation is an absolute contributing factor in the final decision. In other words, the different objectives and limitations influencing the judgment are integrated using fuzzy aggregation operators. Indeed, fuzzy logic is a gifted approach to the combination of multi-criteria judgment $[10,19]$.

In order to combine data by fuzzy logic, a specific approach should be followed. Firstly, crisp data sets should be fuzzified and then, by applying guide-lines, all fuzzy data are fused in a fuzzy ambience. where each element of $X$ is mapped to a value between 0 and 1 . This value, named the membership value or degree of membership, quantifies the grade of membership of the element in $X$ to the fuzzy set $A$. The degree of membership varies from 0 , where ' $u$ ' is not a member of combination ' $\mathrm{A}$ ', to 1 , where ' $\mathrm{u}$ ' completely relates to combination 'A' $[17,18]$. Finally, fuzzy outputs convert to crisp outputs using a membership function. Figure 4 illustrates the steps used in this study to combine data.

In order to obtain satisfactory results, fuzzy logic has various tools to deal with fuzzy sets including S-norms, T-norms, etc. each of which has its specific application. In this study, Snorms are used as fusion rules in fuzzy logic to combine two data sets [10]. In fact, S-norm functions are a particular form of top values sum. Owing to the fact that the aim of this study is to combine data in the way that two data sets support each other, S-norms are selected as fusion rules. Though there are multiple S-norm versions of fuzzy fusion encompassing Einstein, Yager, Domby, Algebraic and Dubois; The Yager version is the one utilized in this study. This kind of S-norm has been employed in other works and has outstanding performance in fusing information [20]. Eq. (17) describes theYager version of fuzzy fusion.

$$
S_{\omega}(a, b)=\min \left[1,\left(a^{\omega}+b^{\omega}\right)^{\frac{1}{\omega}}\right], \quad \text { For } \omega \in(0, \infty)
$$




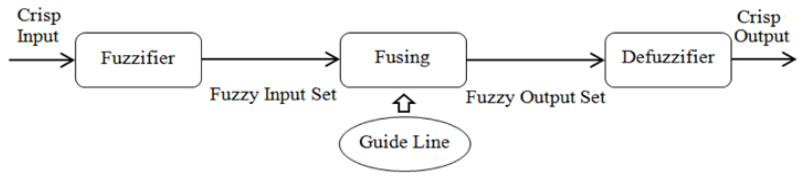

FIG. 4 The process of fuzzy combination.

\section{RESULTS AND DISCUSSION}

\subsection{Using Data Fusion Method to compare the Performance of DOP min and DOPave}

As discussed in Section 2.2, using either $D O P_{\text {min }}$ or $D O P_{\text {ave }}$ as control signals in a DGD monitoring system has a positive and a negative aspect: $D O P_{\text {min }}$ is more sensitive (as a positive factor) to DGD variations, while there is more ambiguity in DGD monitoring (as a negative factor) when $D O P_{\min }$ is used as a control signal compared with $D O P_{\text {ave }}$. In this case, determining the control signal that has a better performance in a DGD monitoring system is challenging. As the data fusion method has a promising effect in similar situations in which decision making is difficult, it is worth applying it in this case. The stages of employing the data fusion approach to evaluate and compare the performance of $D O P_{\min }$ and $D O P_{\text {ave }}$ in a DGD monitoring system are shown in Figure 5. As is shown in Figure 5, first $\triangle \mathrm{MDD}$ and $\triangle \mathrm{SMDD}$ are measured at different points. Then, the data obtained are prepared at the preparation data stage. At this stage, some proper processes including data normalization between 0 and 1 for both $\triangle \mathrm{MDD}$ and $\triangle$ SMDD and inverting $\triangle$ SMD are imposed on the data. Afterwards, the prepared data are fused in the fusion centre by using a suitable fuzzy combinator, Yager version of fuzzy fusion is employed in the fusion centre. Finally, the outputs of the fusion centre named $D O P_{\min }$ Combined Data are obtained. The similar processes are imposed on $\triangle \mathrm{ADD}$ and $\triangle \mathrm{SADD}$ to obtain the $D O P_{\text {ave }}$ Combined Data. Then, the Combined Data of $D O P_{\min }$ and $D O P_{\text {ave }}$ can be properly compared, in order to indicate more efficient control signal. The results obtained are shown in Figure 6, which is the curve of Combined Data vs. DGD/T. Comparison between the values of $D O P_{\text {min }}$ and $D O P_{\text {ave }}$ Combined Data demonstrates that $D O P_{\text {ave }}$ is a more efficient control signal than $D O P_{\text {min }}$ in DGD monitoring system, because the values of Combined Data of $D O P_{\text {ave }}$ are greater than that of $D O P_{\min }$ for different values of DGD/T. This means that, in weighting the pros and cons of each control signal, $D O P_{\text {ave }}$ outperforms $D O P_{\text {min }}$ in a DGD monitoring system. It can therefore be interpreted that using $D O P_{\text {ave }}$ as a control signal in a PMDC system leads to achieving better results in lowering outage probability, as compared with using $D O P_{\text {min }}$ since a PMDC system with $D O P_{\text {ave }}$ as a control signal can track DGD variations more effectively. Therefore, PMD effects can be further mitigated and, as a result, the outage probability can be further lowered. It can also be concluded from Figure 6 that the performance of $D O P_{\text {ave }}$ and $D O P_{\text {min }}$ in monitoring DGD improves when DGD/T values varies. From 0 to 0.3 . Furthermore, as $\mathrm{DGD} / \mathrm{T}$ values increase from 0.3 , the efficiency of the performance of DOPmin and DOPave as control signals in a DGD monitoring system gradually decreases. Moreover, for a DGD/T value around 0.5 , the performance of these two control signals becomes very similar. Additionally, according to the Figure 2, the range of DGD

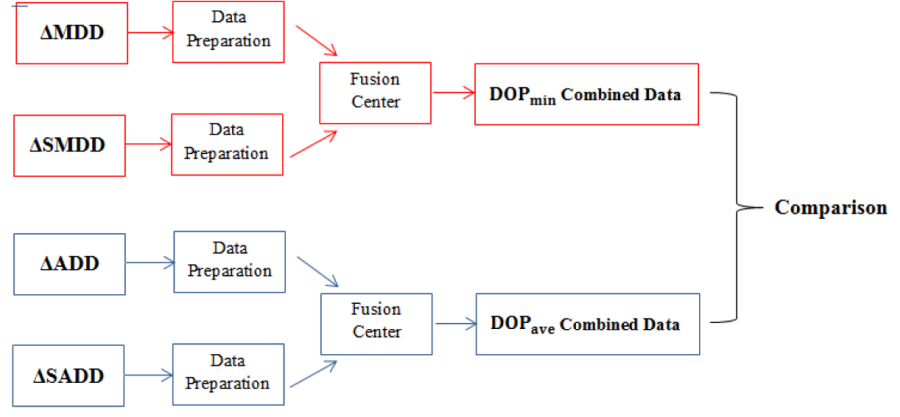

FIC. 5 The stages of applying the data fusion method to compare the performance of $D O P_{\min }$ and $D O P_{\text {ave }}$ in a DGD monitoring system.

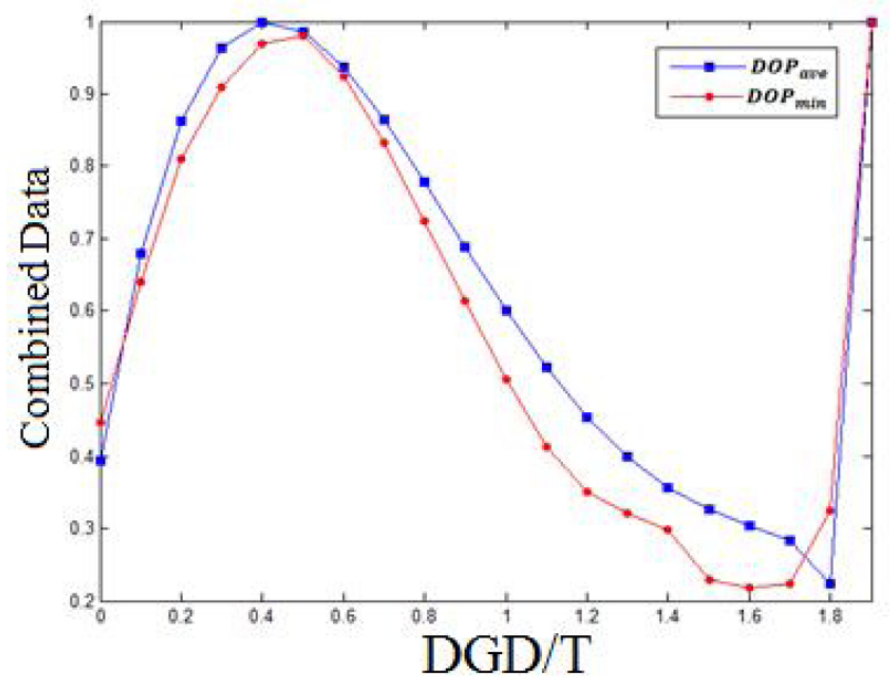

FIC. 6 The Combined Data variations vs. DGD/T variations curve for $D O P_{\min }$ and $D O P_{a v e}$ in the presence of all-order PMD.

monitoring is limited because of the insensitivity of DOP to $\mathrm{DGD} / \mathrm{T}$ values greater than 1.8. Therefore, in Figure 6, the Combined Data values for DGD/T values greater than 1.8 are invalid. In the following section, $D O P_{\min }$ and $D O P_{\text {ave }}$ are employed in a feed-forward first-order PMDC system - in order to test the reliability of the results obtained from the data fusion method and compare the performance of $D O P_{\min }$ and $D O P_{a v e}$ in lowering the outage probability.

\subsection{Testing Results obtained from the Data Fusion in a first-order PMDC system}

The higher-order PMD vectors are the coefficients in the Taylor expansion of the measured output PMD vector $(\vec{\tau}(\omega))$ around the carrier frequency $\omega_{0}$ and are given by [21]:

$$
\begin{aligned}
\vec{\tau}(\omega)= & \vec{\tau}\left(\omega_{0}\right)+\vec{\tau}_{\omega} \Delta \omega+\frac{1}{2} \vec{\tau}_{\omega \omega} \Delta^{2}+\ldots \\
& +\frac{1}{n !} \vec{\tau}^{(n)} \Delta \omega^{n}
\end{aligned}
$$

where $\vec{\tau}_{\omega}$ is the second-order PMD vector, $\vec{\tau}_{\omega \omega}$ is thirdorder PMD vector and so on. Moreover, $\omega=\omega_{0}+\Delta \omega$ and $\Delta \omega$ is the frequency deviation from the carrier. Since the firstorder PMD vector $\vec{\tau}\left(\omega_{0}\right)$ is essentially constant within the bandwidth of the PSP, and higher-order PMD effects are usually negligible when the signal bandwidth is smaller than $\Delta \omega_{P S P}$, first-order PMD can be considered as a special case in 


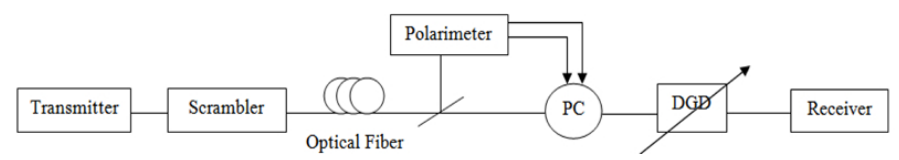

FIG. 7 Block diagram of a feed-forward first-order PMD compensation system with a variable delay line.

which other terms related to the higher-order PMD in the Taylor expansion are neglected. Hence, the results obtained from the data fusion method can be tested in a first-order PMDC system as a special case that is simpler than higher-order ones. A simple feed-forward PMDC system, as presented in [22] and shown in Figure 7, that is capable of compensating firstorder PMD is selected - in order to evaluate and compare the performance of $D O P_{\text {ave }}$ and $D O P_{\text {min }}$ as control signals in mitigating the outage probability. In this system, The SOP of an optical source is rapidly scrambled before launching light into the optical link. At the end of the link, a polarimeter is applied to analyze and measure the output SOPs and corresponding DOPs during PSP of each period of scrambling. Then, while the compensator remains stable over the scrambling period (which is a length of time around millisecond [23]), a feedforward control signal that is based on $D O P_{\text {min }}$ (or $D O P_{\text {ave }}$ ) is computed over a defined scrambling period and then, it adjusts the compensator for the next period in agreement with the output PSP and DGD. By applying the DOP vs. DGD curve shown in Figure 2, DGD can be estimated as a function of $D O P_{\min }$ (or $D O P_{a v e}$ ). For calculation of the outage probability of the system, the bit error rate (BER) can be calculated by using Gaussian statistics [24] and an outage is defined whenever BER $\geq 10^{-12}$ [25]. For analysis of the system, the model of reference [20] has been used - and PRBS sequences of super-Gaussian NRZ pulses with a word length of $2^{5}-1$ in a $40-\mathrm{Gb} / \mathrm{s}$ transmission system are launched into the fiber optic link. The output sequences with an EDFA-preamplifier (noise figure $3 \mathrm{~dB}$ and a $1.3 \mathrm{~nm}$ optical filter) at the receiver are detected when there is a fifth-order Bessel low-pass filter with 24-GHz bandwidth at the receiver. The BER can then be computed in a large number of fiber realizations with a specific mean DGD. Finally, by taking average over the BER outages the outage probability of the fiber optic link is obtained.

The results are shown in Figure 8 and it can be concluded that the compensation system in which $D O P_{\text {ave }}$ is used as control signal better performs in lowering outage probability compared with the compensation system with $D O P_{\text {min }}$ as control signal. Also, as shown in Figure 6, when the average DGD/T increases from 0.3 , the efficiency of the performance of both control signals in the lowering outage probability gradually decreases. Thus, the results previously obtained by the data fusion method are confirmed by the introduced PMDC system.

\section{CONCLUSIONS}

PMD is a potential limiting factor in long-haul high-speed optical communications. Due to the stochastic nature of PMD, an adaptive PMDC system which can dynamically track and mitigate its effects is needed. One of the most important parts of

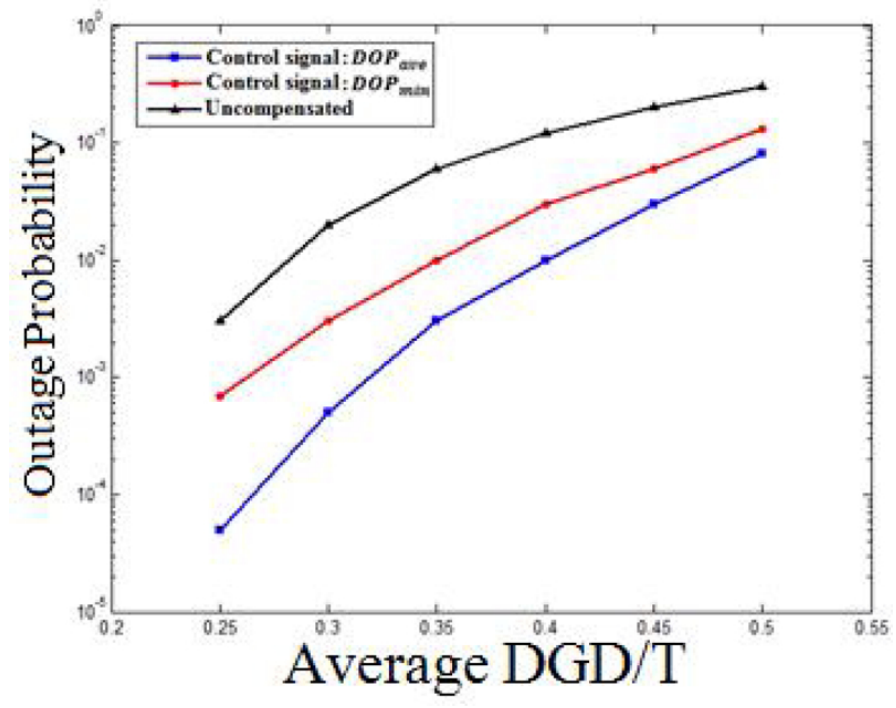

FIG. 8 Outage probability vs. DGD/T curve in a feed-forward first-order PMD compensation system (with a variable delay line) using $D O P_{\min }$ and $D O P_{\text {ave }}$ as control signals.

an adaptive PMDC system is the monitoring system since selecting an efficient control signal in a PMD monitoring system can improve the performance of PMDC system properly. In this paper, the performance of $D O P_{\min }$ and $D O P_{\text {ave }}$ as control signals in a DGD monitoring system was analyzed and evaluated when there existed all-order PMD. Results showed that $D O P_{\text {min }}$ is more sensitive than $D O P_{\text {ave }}$ to DGD variations, but there is more ambiguity in DGD monitoring when $D O P_{\text {min }}$ is used as a control signal. Then, since each of these two control signals had a negative and a positive aspect to be efficient in a DGD monitoring system, decision making on selecting more efficient control signal and evaluating and comparing their performance are challenging. As a result, we have used a data fusion method that fused two features of each control signal (the amount of sensitivity and the ambiguity in DGD monitoring). By applying this method, we could evaluate and compare the performance of these control signals while considering both their negative and positive aspects in a DGD monitoring system - even before employing them in a PMDC system. The outcomes of the data fusion method have demonstrated that generally $D O P_{\text {ave }}$ is a more efficient control signal than $D O P_{\text {min }}$ in a DGD monitoring system, the performance of both control signals becomes almost similar at a DGD/T value around 0.5 - and, as DGD/T values increase from 0.3 , the their efficiency gradually decreases. Finally, we used mentioned control signals in a feed-forward first-order PMDC to investigate the reliability of data fusion outcomes. The results have shown that data fusion is a worthy software approach for employing more than one feature of control signals to compare their performance, before testing them in a real compensation system.

In practice, we can evaluate and compare the performance of control signals in different values of average DGD/T of the fiber optic links simultaneously, and decide which control signal will be more useful in reducing PMD by using the Combined Data vs. DGD/T curve. In fact, in this study, we have shown that the data fusion method can be used to predict the performance of two identified control signals in PMDC sys- 
tems. The advantage of using the data fusion method is that it makes it possible to obtain a general view of the performance of two chosen control signals in different PMDC systems. Indeed, the data fusion method has the capability of being a promising method to enhance evaluation of the efficiency of control signals. This approach can be used in electronic and optical signal processing and can facilitate evaluating and comparing the performance of different control signals. However, before this method can enjoy widespread use, various investigations and researches must be carried out.

\section{References}

[1] H. Miao, A. M. Weiner, L. Mirkin, and P. J. Miller, "All-order polarization-mode dispersion (PMD) compensation via virtually imaged phased array (VIPA)-based pulse shaper," IEEE Photonics Technol. Lett. 20, 545-547 (2008).

[2] L. Xu, H. Miao, and A. M. Weiner, "All-order polarization-modedispersion(PMD) compensation at $40 \mathrm{~Gb} / \mathrm{s}$ via hyperfine resolution optical pulse shaping," IEEE Photonics Technol. Lett. 22, 1078-1080 (2010).

[3] J. T. Rahn, H. Sun, K. T. Wu, and B. E. Basch, "Real-Time PMD Tolerance Measurements of a PIC-Based $500 \mathrm{~Gb} / \mathrm{s}$ Coherent Optical Modem," J. Lightwave Technol. 30, 2907-2912 (2012).

[4] H. Miao, A. M. Weiner, L. Mirkin, and P. J. Miller, "Broadband allorder polarization mode dispersion compensation via wavelengthby-wavelength Jones matrix correction," Opt. Lett. 32, 2360-2362 (2007).

[5] A. Weiner, M. Akbulut, S. X. Wang, and P. J. Miller, “Optical parallel processing approach to all-order PMD compensation," in Proceedings of 2006 Optical Fiber Communication Conference 1 (Optical Society of America, Anaheim, 2006).

[6] C. Floridia, G. C. C. P. Simões, M. M. Feres, and M. A. Romero, "Simultaneous optical signal-to-noise ratio and differential group delay monitoring based on degree of polarization measurements in optical communications systems," Appl. Optics 51, 3957-3965 (2012).

[7] A. E. Willner, S. M. R. M. Nezam, L. Yan, Z. Pan, and M. C. Hauer, "Monitoring and control of polarization-related impairments in optical fiber systems," J. Lightwave Technol. 22, 106-125 (2004).

[8] N. Kikuchi, "Analysis of signal degree of polarization degradation used as control signal for optical polarization mode dispersion compensation," J. Lightwave Technol. 19, 480-486 (2001).

[9] R. Cigliutti, A. Galtarossa, M. Giltrelli, D. Grosso, A. W. R. Leitch, L. Palmieri, S. Santoni, et al., "Design, estimation and experimental validation of optical polarization mode dispersion compensator in $40 \mathrm{Gbit} / \mathrm{s}$ NRZ and RZ optical systems," Opt. Fiber Technol. 15, 242-250 (2009).

[10] A. Habibalahi, and M. S. Safizadeh, "Pulsed eddy current and ultrasonic data fusion applied to stress measurement," Meas. Sci. Technol. 25, 055601 (2014).
[11] A. Orlandini, and L. Vincetti, "A simple and useful model for Jones matrix to evaluate higher order polarization-mode dispersion effects," IEEE Photonics Technol. Lett. 13, 1176-1178 (2001).

[12] C. Brosseau, Fundamentals of polarized light: a statistical optics approach (Wiley, New York, 1998).

[13] S. M. Nezam, J. E. McGeehan, and A. E. Willner, "Theoretical and experimental analysis of the dependence of a signal's degree of polarization on the optical data spectrum," J. Lightwave Technol. 22, 763-772 (2004).

[14] M. Safari, and A. A. Shishegar, "Analysis of degree of polarization as a control signal in PMD compensation systems aided by polarization scrambling," J. Lightwave Technol. 26, 2865-2872 (2008).

[15] G. J. Foschini, L. E. Nelson, R. M. Jopson, and H. Kogelnik, "Statistics of second-order PMD depolarization," J. Lightwave Technol. 19, 1882-1886 (2001).

[16] P. Y. Ekel, M. Menezes, and F. H. S. Neto, “Decision making in a fuzzy environment and its application to multicriteria power engineering problems," Nonlinear Anal-Hybri. 1, 527-536 (2007).

[17] L. A. Zadeh, "Fuzzy sets," Information and control," 8, 338-353 (1965).

[18] L. X. Wang, A course in fuzzy systems (Prentice-Hall press, New Jersey, 1999).

[19] T. G. dos Santos, B. S. Silva, P. dos Santos Vilaça, L. Quintino, and J. M. C. Sousa, "Data fusion in non destructive testing using fuzzy logic to evaluate friction stir welding," Welding Int. 22, 826-833 (2008).

[20] L. A. Klein, Sensor and data fusion concepts and applications (Society of Photo-Optical Instrumentation Engineers (SPIE, Bellingham, 1993).

[21] H. Chen, R. Jopson, and H. Kogelnik, "On the bandwidth of higherorder polarization-mode dispersion: the Taylor series expansion," Opt. Express 11, 1270-1282 (2003).

[22] P. C.Chou, J. M. Fini, and H. Haus, "Real-time principal state characterization for use in PMD compensators," IEEE Photonics Technol. Lett. 13, 568-570 (2001).

[23] A. E. Willner, S. M. R. M. Nezam, L. Yan, Z. Pan, and M. C. Hauer, "Monitoring and control of polarization-related impairments in optical fiber systems," J. Lightwave Technol. 22, 106-125 (2004).

[24] G. P. Agrawal, Fiber-optic communication systems (Wiley, New York, 1997).

[25] H. Sunnerud, C. Xie, M. Karlsson, R. Samuelsson, and P. A. Andrekson, "A comparison between different PMD compensation techniques," J. Lightwave Technol. 20, 368-378 (2002). 